\title{
On certain K-equivalent birational maps
}

\author{
Duo Li
}

\begin{abstract}
We study K-equivalent birational maps which are resolved by a single blowup. Examples of such maps include standard flops and twisted Mukai flops. We give a criterion for such maps to be a standard flop or a twisted Mukai flop. As an application, we classify all such birational maps up to dimension 5 .
\end{abstract}

\section{Contents}

1. Introduction 1

2. Proof of main results 4

3. Classifications when $\operatorname{dim} X \leq 5 \quad 10$

References 14

1. Introduction

In this article, all the varieties are defined over complex numbers and are assumed to be projective and smooth. By projective bundle, we mean that there exists a locally free sheaf $\mathcal{E}$ s.t. $\mathbb{P}(\mathcal{E})=\operatorname{Proj}\left(\operatorname{Sym}\left(\mathcal{E}^{\vee}\right)\right)$. By $\mathbb{P}^{n}$ fibration $\alpha: X \rightarrow Y$, we mean that $\alpha^{-1}(y)$ is isomorphic to $\mathbb{P}^{n}$ for every closed point $y \in Y$.

In this article, we address the study of the birational map $\theta: X-->X^{+}$ which is K-equivalent and can be resolved by a single blowup. We call $\theta$ a birational map of Simple Type. To be concrete, there is a closed smooth subvariety $P$ of $X$ (resp. $P^{+}$a closed smooth subvariety of $X^{+}$) and the blowup $\varphi: B l_{P}(X) \rightarrow X$ along $P\left(\right.$ resp. $\varphi^{+}: B l_{P}^{+}\left(X^{+}\right) \rightarrow X^{+}$along $P^{+}$) satisfies that:

(1) $B l_{P}(X) \simeq B l_{P^{+}}\left(X^{+}\right)$(we denote this variety by $\left.\widetilde{X}\right)$. 
(2) $\theta \circ \varphi=\varphi^{+}$, i.e., the diagram

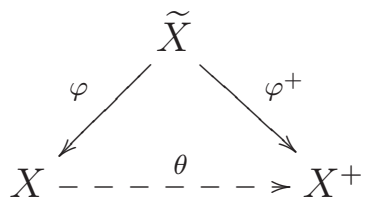

is commutative.

(3) $\varphi^{*}\left(K_{X}\right)=\varphi^{+*}\left(K_{X^{+}}\right)$in $\operatorname{Pic}(\tilde{X})$.

(4) $\theta$ is not an isomorphism.

Actually, we know two concrete constructions of birational maps of simple type as follows:

1. standard flop (over a base $C$ )(In article [8], standard flops are called ordinary flops instead):

Suppose that there is a closed subvariety $P$ of $X$ satisfies that $\pi: P=$ $\mathbb{P}_{C}(\mathcal{E}) \rightarrow C$ is a projective bundle. The normal bundle of $P$ satisfies that $N_{P / X}=\pi^{*} \mathcal{E}^{+} \otimes \mathcal{O}(-1)$ where $\mathcal{E}^{+}$is a vector bundle over $C$ and $\mathcal{O}(1)$ is the tautological bundle for $\mathbb{P}_{C}(\mathcal{E})$. Then we consider the blowup of $X$ along $P$. Let $P^{+}=\mathbb{P}_{C}\left(\mathcal{E}^{+}\right)$. We denote $\pi^{+}: P^{+} \rightarrow C$ the projective bundle morphism, then the exceptional divisor $E \simeq P \times_{C} P^{+}$. For any fibre $F$ of $p^{+}: E \rightarrow P^{+}$, by a simple calculation, we have that $\left.\mathcal{O}_{E}(E)\right|_{F} \simeq \mathcal{O}(-1)$ (see Chapter 11, [5] or Section 1, [8]). By Fujiki-Nakano's criterion(see [2] or Remark 11.10, [5]), there is a blow-down $\varphi^{+}: \widetilde{X} \rightarrow X^{+}$compatible with $p^{+}$. Hence we obtain a birational map $\theta: X \rightarrow X^{+}$, we call $\theta$ a standard flop over $C$.

2. twisted Mukai flop (over a base $C$ ):

Suppose that there is a closed subvariety $P$ of $X$ satisfies that $\pi: P=$ $\mathbb{P}_{C}(\mathcal{E}) \rightarrow C$ is a projective bundle. The normal bundle of $P$ satisfies that $N_{P / X}=\Omega_{P / C} \otimes \pi^{*} \mathcal{L}$ for some line bundle $\mathcal{L}$ of $C$. Then we consider the blowup of $X$ along $P$. Let $P^{+}=\mathbb{P}_{C}\left(\mathcal{E}^{\vee}\right)$. We denote $\pi^{+}: P^{+} \rightarrow C$ the projective bundle morphism, then the exceptional divisor $E \simeq \mathbb{P}_{P}\left(\Omega_{P / C}\right)$ is a prime divisor of $P \times_{C} P^{+}$, hence there is an induced projective bundle structure $p^{+}: E \rightarrow P^{+}$. For any fibre $F$ of $p^{+}$, by a simple calculation, we have that $\left.\mathcal{O}_{E}(E)\right|_{F} \simeq \mathcal{O}(-1)$ (see Chapter 11, [5] or Section 6 of [8]). By Fujiki-Nakano's criterion(see [2] or Remark 11.10, [5]), there is a blow-down $\varphi^{+}: \widetilde{X} \rightarrow X^{+}$compatible with $p^{+}$. Hence we obtain a birational map $\theta: X \rightarrow X^{+}$, we call $\theta$ a twisted Mukai flop over $C$, if $\mathcal{L}$ is a trivial line bundle, we call $\theta$ a Mukai flop for short. 
Remark 1.1. In the above constructions, $X^{+}$is not necessarily projective. But it is reasonable to assume that there exists a flopping contraction $\beta: X \rightarrow$ $\bar{X}$ compatible with $\pi: P \rightarrow C$. Under this assumption, $X^{+}$can be proved projective. For details, see Proposition 1.3 and Proposition 6.1, [8].

There is a natural question: are all birational maps of simple type either standard flops or twisted Mukai flops? Actually, we construct a new simple type birational map in Example 3.8 and one of the main results of our article is:

Theorem 1.2. A birational map of simple type, if $\operatorname{dim} X \leq 5$, is either a standard flop, a twisted Mukai flop or a flop as in Example 3.8.

For properties about standard flops and twisted Mukai flops, we use [5] and [8] as our main references. We note that in articles [3] and [8], motivic and quantum invariance under a standard flop or a twisted Mukai flop was studied, we hope this article could offer useful information to further study in that direction.

Now let us state the structure of this article: first, we prove that $\varphi$ and $\varphi^{+}$ share a common exceptional divisor $E$ and $\operatorname{dim} P=\operatorname{dim} P^{+}$. Then we generalise E. Sato' classification results about varieties which admits two different projective bundle structures to a relative version. The main result of this article is Theorem 2.6, we prove that a birational map of simple type is a standard flop or a twisted Mukai flop if and only if $P$ and $P^{+}$are projective bundles over a common variety. As an application of our main result, we classify simple type birational maps when $\operatorname{dim} X \leq 5$, here recent results of [6] and [17] play important roles in our classification.

Acknowledgments. The author is very grateful to Professor Baohua Fu for his support, encouragement and stimulating discussions over the last few years. The author is very grateful to Professor Chin-Lung Wang for his helpful suggestions and discussions. The author wishes to thank Yang Cao, Yi Gu, Wenhao Ou, Xuanyu Pan, Lei Zhang for useful discussions and thank Professor Xiaokui Yang for his support and encouragement. 


\section{Proof of main results}

Let us start with the following observation, which shows that $\varphi$ and $\varphi^{+}$share a common exceptional divisor $E$, hence $E$ admits two projective bundle structures. We will repeatedly use this fact in the rest of our article.

Lemma 2.1. The blowups $\varphi$ and $\varphi^{+}$share a common exceptional divisor $E$ and $\operatorname{dim} P=\operatorname{dim} P^{+}$

Proof. Let $k=\operatorname{codim}(P, X)$ and $k^{+}=\operatorname{codim}\left(P^{+}, X^{+}\right)$, then $k, k^{+} \geq 2$. Since $\varphi$ and $\varphi^{+}$are blowups, the corresponding exceptional divisors are $E$ and $E^{+}$, we have the following two equations:

(1) $\varphi^{*} K_{X}=K_{\tilde{X}}-(k-1) E$

(2) $\varphi^{+*} K_{X^{+}}=K_{\tilde{X}}-\left(k^{+}-1\right) E^{+}$.

Since $\varphi^{*}\left(K_{X}\right)=\varphi^{+*}\left(K_{X^{+}}\right), E$ is numerically equivalent to $\frac{k^{+}-1}{k-1} E^{+}$, where $\frac{k^{+}-1}{k-1}>0$.

Since $E$ is a projective bundle over $P$, for any point $p \in P$, the fiber $F_{p}$ of $\varphi$ is isomorphic to $\mathbb{P}^{k-1}$. For any line $C$ in $F_{p} \simeq \mathbb{P}^{k-1}, C \cdot E=\left.\operatorname{deg} \mathcal{O}_{\tilde{X}}(E)\right|_{C}=$ $\operatorname{deg} \mathcal{O}(-1)=-1$. As $p$ varies, these negative lines in $F_{p}$ cover $E$.

If $E \neq E^{+}$, then there exists a line $C$ in $F_{p_{0}}$ for some $p_{0} \in P$ such that $C$ is not contained in $E^{+}$. So $C \cdot E=C \cdot\left(\frac{k^{+}-1}{k-1}\right) E^{+} \geq 0$, contradicts to $C \cdot E<0$.

Then $E=E^{+}$and $k^{+}=k$.

By Propostion 1.14, [1], $\left.\varphi\right|_{E}$ and $\left.\varphi^{+}\right|_{E}$ are non-isomorphic fibrations. There is a lower bound for the dimension of $P$.

Lemma 2.2. $2 \operatorname{dim} P+1 \geq \operatorname{dim} X$.

Proof. The exceptional divisor $E$ has two projective bundle structures over $P$ and $P^{+}$. We denote these two projective bundle morphisms in the following diagram:

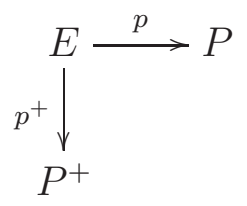

where $p=\left.\varphi\right|_{E}$ and $p^{+}=\left.\varphi^{+}\right|_{E}$. Since fibers of different extremal ray contractions can meet only in points, $\left(p, p^{+}\right): E \longrightarrow P \times P^{+}$is a finite morphism to its image. So $\operatorname{dim} E \leq 2 \operatorname{dim} P$, which means that $\operatorname{dim} X \leq 2 \operatorname{dim} P+1$. 
Now recall E.Sato's classification about varieties which admit two projective bundle structures (See Theorem A in [14]).

Theorem 2.3. If E has two projective bundle structures over projective spaces:

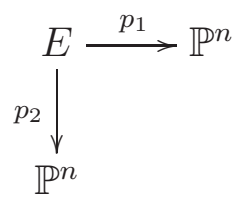

then $E$ is isomorphic to either $\mathbb{P}^{n} \times \mathbb{P}^{n}$ (in this case, $\left.\operatorname{dim} E=2 n\right)$ or $\mathbb{P}_{\mathbb{P}^{n}}\left(\Omega_{\mathbb{P}^{n}}\right)$ (in this case, $\operatorname{dim} E=2 n-1$ ).

Remark 2.4. We now sketch the proof of E.Sato's theorem in the case of $\operatorname{dim} E=2 n-1$. First, it can be proved that $\Phi=\left(p_{1}, p_{2}\right): E \longrightarrow \mathbb{P}^{n} \times \mathbb{P}^{n}$ is a closed immersion. Then $\Phi(E)$ is a Cartier divisor in $\mathbb{P}^{n} \times \mathbb{P}^{n}$, we denote the defining equation of $\Phi(E)$ by $F\left(X_{0} \cdots, X_{n} ; Y_{0} \cdots, Y_{n}\right)$. The key point is $F$ is a homogeneous polynomial of bidegree $(1,1)$. Moreover, it can be shown that after performing suitable linear transforms of $\left(X_{0} \cdots, X_{n}\right)$ and $\left(Y_{0} \cdots, Y_{n}\right), F$ is of the form $F=\sum_{i=0}^{n} X_{i} \cdot Y_{i}$. If we view $\mathbb{P}_{\mathbb{P}^{n}}\left(\Omega_{\mathbb{P}^{n}}\right)$ as a closed subvariety of $\mathbb{P}^{n} \times\left(\mathbb{P}^{n}\right)^{*}$ by the Euler sequence:

$$
0 \rightarrow \Omega_{\mathbb{P} n} \rightarrow \mathcal{O}(-1)^{n+1} \rightarrow \mathcal{O} \rightarrow 0
$$

then $E$ has the same defining equation as that of $\mathbb{P}_{\mathbb{P}^{n}}\left(\Omega_{\mathbb{P}^{n}}\right)$.

Now we generalise E.Sato's result to a relative version.

Theorem 2.5. Suppose that E admits two projective bundle structures:

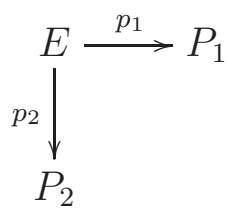

If the following conditions are satisfied:

(1) $P_{1}$ and $P_{2}$ are projective bundles over a common variety $C$, i.e. $P_{1}=$ $\mathbb{P}_{C}\left(\mathcal{E}_{1}\right)$ and $P_{2}=\mathbb{P}_{C}\left(\mathcal{E}_{2}\right)$ for some locally free sheaves $\mathcal{E}_{i}$ on $C$.

(2) Let $\pi_{i}: P_{i} \longrightarrow C$ denote the projective bundle morphism, we assume that $\pi_{1} \circ p_{1}=\pi_{2} \circ p_{2}$.

(3) $\operatorname{dim} P_{1}=\operatorname{dim} P_{2}$.

then $E$ is isomorphic to either $P_{1} \times_{C} P_{2}$ or $\mathbb{P}_{P_{1}}\left(\Omega_{P_{1} / C}\right)$. In the first case, $\operatorname{dim} E=$ $2 \operatorname{dim} P_{1}-\operatorname{dim} C ;$ in the second case, $\operatorname{dim} E=2 \operatorname{dim} P_{1}-\operatorname{dim} C-1$. 
Proof. We assume that the rank of $\mathcal{E}_{i}$ is $k+1$ and let $\alpha=\pi_{1} \circ p_{1}$. Then the following diagram :

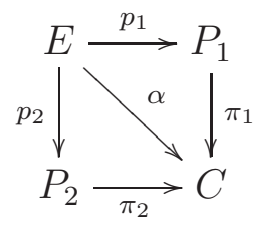

commutes. Since every fiber of $\alpha$ has two projective bundle structures over projective spaces, by Theorem 2.3, $\operatorname{dim} E=2 \operatorname{dim} P_{1}-\operatorname{dim} C$ or $\operatorname{dim} E=$ $2 \operatorname{dim} P_{1}-\operatorname{dim} C-1$.

In the case of $\operatorname{dim} E=2 \operatorname{dim} P_{1}-\operatorname{dim} C$, we aim to show that $E \simeq P_{1} \times_{C} P_{2}$. Note that we have the following commutative diagram:

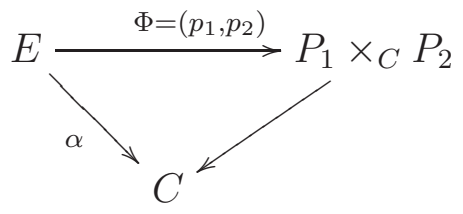

For any $c \in C$, let $E_{c}$ be the fiber of $\alpha$. Then by Theorem 2.3, the restriction of $\Phi$ to $E_{c}$ :

$$
\Phi_{c}: E_{c} \longrightarrow \pi_{1}^{-1}(c) \times \pi_{2}^{-1}(c) \simeq \mathbb{P}^{k} \times \mathbb{P}^{k}
$$

is an isomorphism. So $\Phi$ is an isomorphism.

In the case of $\operatorname{dim} E=2 \operatorname{dim} P_{1}-\operatorname{dim} C-1$, we aim to show that $E \simeq$ $\mathbb{P}_{P_{1}}\left(\Omega_{P_{1} / C}\right)$.

We note that $\Phi=\left(p_{1}, p_{2}\right): E \longrightarrow P_{1} \times_{C} P_{2}$ is a closed immersion. First, $\Phi$ is finite onto its image. Otherwise, there will be a curve contracted by both $p_{i}$. Since for any curve $C$ of $\mathbb{P}^{k}$, deformations of $C$ cover $\mathbb{P}^{k}$, then there is a fibre of $p_{1}$ contracted by $p_{2}$. So by the rigidity lemma, $p_{1}=p_{2}$ as fibrations, which is a contradiction to our assumptions. Also, for an arbitrary $c \in C$, by Theorem $2.3, \Phi_{c}: E_{c} \longrightarrow \pi_{1}^{-1}(c) \times \pi_{2}^{-1}(c) \simeq \mathbb{P}^{k} \times \mathbb{P}^{k}$ is a closed immersion. Moreover, $E_{c}$ is a prime divisor defined by a homogeneous polynomial of bidegree $(1,1)$. Then $\Phi$ is a finite morphism to its image of degree one, so $\Phi$ is a closed immersion.

Let $X=P_{1} \times_{C} P_{2}, q_{i}: X \rightarrow P_{i}$ denote the projection morphism and $f: X \rightarrow C$ be the base morphism. We view $E$ as a prime divisor of $X$, then $\mathcal{O}_{X}(E) \simeq q_{1}^{*} \mathcal{O}(1) \otimes q_{2}^{*} \mathcal{O}(1) \otimes f^{*} \mathcal{L}$ where $\mathcal{O}(1)$ denotes the tautological line bundle of a given projective bundle and $\mathcal{L}$ is a line bundle of $C$. Note that 
$f_{*}\left(\mathcal{O}_{X}(E)\right) \simeq \mathcal{E}_{1}^{\vee} \otimes \mathcal{E}_{2}^{\vee} \otimes \mathcal{L}$. Since $E$ is an effective divisor, there is a non-zero global section of $H^{0}\left(C, f_{*}\left(O_{X}(E)\right)\right)=H^{0}\left(X, \mathcal{O}_{X}(E)\right)$. Then this global section induces a morphism of sheaves $\mathcal{O} \rightarrow \mathcal{E}_{1}^{\vee} \otimes \mathcal{E}_{2}^{\vee} \otimes \mathcal{L}$, hence a linear form $\beta: \mathcal{E}_{1} \otimes \mathcal{E}_{2} \rightarrow \mathcal{L}$

Actually, the linear form $\beta$ is non-degenerate. For any affine open subset SpecA of $C, \Phi(E)$ is a Cartier divisor of $P_{1} \times_{A} P_{2}$. Then $\Phi(E)$ is defined by a single equation $F\left(X_{0} \cdots, X_{k} ; Y_{0} \cdots, Y_{k}\right)=0$ whose coefficients are elements of $A$. For any local sections of $\mathcal{E}_{1}$ and $\mathcal{E}_{2}: e_{1}=\left(x_{0} \cdots, x_{k}\right), e_{2}=\left(y_{0} \cdots, y_{k}\right)$, we have that $\beta\left(e_{1}, e_{2}\right)=F\left(x_{0} \cdots, x_{k} ; y_{0} \cdots, y_{k}\right)$. Since $F$ is a homogeneous polynomial of bidegree $(1,1)$, we can write $\beta$ as :

$$
\beta\left(e_{1}, e_{2}\right)=\left(\begin{array}{lll}
x_{0} & \cdots & x_{k}
\end{array}\right) M\left(\begin{array}{c}
y_{0} \\
\vdots \\
y_{k}
\end{array}\right)
$$

where $M$ is a matrix in $M_{n}(A)$. By Theorem 2.3 and Remark 2.4, $\operatorname{det}(M)$ is invertible in $A_{m}$ for any maximal ideal $m$ of $A$, so $M$ is an invertible matrix, which means that the linear form $\beta(-,-)$ is non-degenerate. So $\mathcal{E}_{1} \simeq \mathcal{E}_{2}^{\vee} \otimes \mathcal{L}$. Since $\mathbb{P}\left(\mathcal{E}_{2} \otimes \mathcal{L}^{-1}\right) \simeq \mathbb{P}\left(\mathcal{E}_{2}\right)$, in what follows we assume that $\mathcal{E}_{1} \simeq \mathcal{E}_{2}^{\vee}$.

By the relative Euler sequence $0 \rightarrow \Omega_{P_{1} / C} \rightarrow \pi_{1}^{*}\left(\mathcal{E}_{1}^{\vee}\right)(-1) \rightarrow \mathcal{O} \rightarrow 0$, $\mathbb{P}_{P_{1}}\left(\Omega_{P_{1} / C}\right)$ is a closed subvariety of $P_{1} \times_{C} P_{2}$. As closed subvarieties of $P_{1} \times_{C} P_{2}$, on any affine piece of $C, E$ and $\mathbb{P}_{P_{1}}\left(\Omega_{P_{1} / C}\right)$ are defined by the same equation $\sum X_{i} \cdot Y_{i}=0$, so $E \simeq \mathbb{P}_{P_{1}}\left(\Omega_{P_{1} / C}\right)$.

Now we assume that $P$ and $P^{+}$are projective bundles over a common variety $C$, i.e. $P \simeq \mathbb{P}_{C}(\mathcal{E})$ and $P^{+} \simeq \mathbb{P}_{C}\left(\mathcal{E}^{+}\right)$where $\mathcal{E}, \mathcal{E}^{+}$are locally free sheaves of rank $n+1$ on $C, \pi: P=\mathbb{P}_{C}(\mathcal{E}) \longrightarrow C$ (resp. $\pi^{+}: P^{+}=\mathbb{P}_{C}\left(\mathcal{E}^{+}\right) \longrightarrow C$ ) is the projective bundle morphism. We let $p=\left.\varphi\right|_{E}$ and $p^{+}=\left.\varphi^{+}\right|_{E}$.

We summarise all the morphisms of our problem in the following diagram:

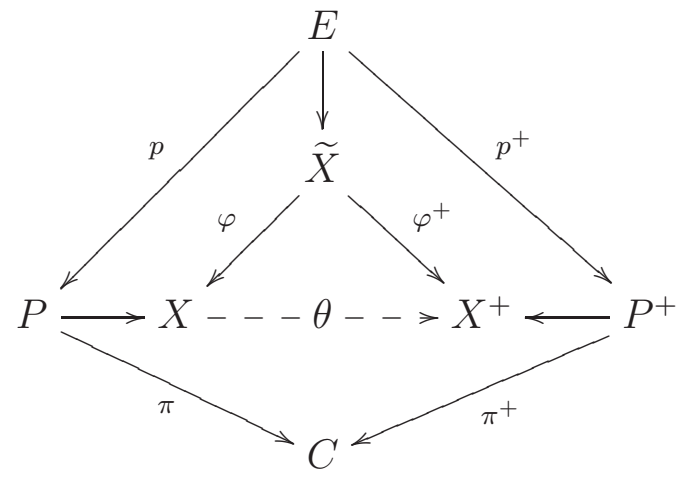


Theorem 2.6. Suppose that $\theta:(X, P) \rightarrow\left(X^{+}, P^{+}\right)$is a birational map of simple type, where $(P, \pi)$ and $\left(P^{+}, \pi^{+}\right)$are projective bundles over a common variety $C$ and $\pi \circ p=\pi^{+} \circ p^{+}$. Then $\theta$ is a standard flop or a twisted Mukai flop over base $C$.

Proof. Since $E$ has two projective bundle structures over $P$ and $P^{+}$, by Theorem 2.5, $E \simeq P \times_{C} P^{+}$or $E \simeq \mathbb{P}_{P}\left(\Omega_{P / C}\right)$.

Assume first that $E \simeq P \times_{C} P^{+}$. Since $E \simeq \mathbb{P}_{P}\left(\pi^{*} \mathcal{E}^{+}\right)$and $E \simeq \mathbb{P}_{P}\left(N_{P / X}\right)$, we can assume that $N_{P / X}=\pi^{*} \mathcal{E}^{+} \otimes \mathcal{L}$ where $\mathcal{L}$ is an invertible sheaf of $P$. Suppose that $\mathcal{L}=\pi^{*} \mathcal{L}^{\prime} \otimes \mathcal{O}(-d)$, where $\mathcal{O}(1)$ is the tautological line bundle of $\pi: P \rightarrow C$ and $\mathcal{L}^{\prime}$ is a line bundle of $C$. Since $\mathbb{P}_{C}\left(\mathcal{E}^{+}\right)=\mathbb{P}_{C}\left(\mathcal{E}^{+} \otimes \mathcal{L}^{\prime}\right)$, we can assume that $N_{P / X}=\pi^{*} \mathcal{E}^{+} \otimes \mathcal{O}(-d)$. We aim to show that $d=1$.

Since $\varphi$ is a blowup, $\left.\omega_{E} \simeq \varphi^{*} \omega_{X}\right|_{E} \otimes \mathcal{O}_{E}((n+1) E)$ where $n+1=\operatorname{codim}(P, X)$. Let $h=p \circ \pi$. We now calculate $\left.\left.\varphi^{*} \omega_{X}\right|_{E} \simeq p^{*} \omega_{X}\right|_{P}$ as follows:

$$
\left.\varphi^{*} \omega_{X}\right|_{E} \simeq p^{*} \omega_{P} \otimes\left(\operatorname{det} N_{P / X}\right)^{-1} \simeq p^{*} \omega_{P} \otimes h^{*}\left(\operatorname{det} \mathcal{E}^{+}\right)^{-1} \otimes p^{*} \mathcal{O}((n+1) d) .
$$

So $\omega_{E} \simeq p^{*} \omega_{P} \otimes h^{*}\left(\operatorname{det} \mathcal{E}^{+}\right)^{-1} \otimes p^{*} \mathcal{O}((n+1) d) \otimes \mathcal{O}_{E}((n+1) E)$. For any fiber $F \simeq \mathbb{P}^{n}$ of $p^{+}, h(F)=c$ for some point $c \in C$. The morphism $p$ maps $F$ isomorphically to $\pi^{-1}(c)=\mathbb{P}^{n}$, which is illustrated as follows:

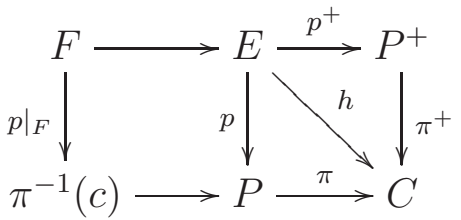

Since $\left.\mathcal{O}_{E}(E)\right|_{F} \simeq \mathcal{O}(-1)$, then we have that

$\left.\left\{p^{*} \omega_{P} \otimes h^{*}\left(\operatorname{det} \mathcal{E}^{+}\right)^{-1} \otimes p^{*} \mathcal{O}((n+1) d) \otimes \mathcal{O}_{E}((n+1) E)\right\}\right|_{F} \simeq \mathcal{O}((n+1)(d-2))$.

We know that $\left.\omega_{E}\right|_{F} \simeq \mathcal{O}(-n-1)$, so $d=1$ which means that $N_{P / X} \simeq$ $\pi^{*} \mathcal{E}^{+} \otimes \mathcal{O}(-1)$ and $\theta$ is a standard flop over $C$.

Assume now that $E \simeq \mathbb{P}_{P}\left(\Omega_{P / C}\right)$. By Theorem 2.5, we know that $\mathcal{E} \simeq \mathcal{E}^{+\vee}$ and $E \hookrightarrow P \times_{C} P^{+}$is a closed immersion. Since $E \simeq \mathbb{P}_{P}\left(N_{P / X}\right)$, we can assume that $N_{P / X}=\Omega_{p / C} \otimes \mathcal{L}$ where $\mathcal{L}$ is an invertible sheaf of $P$. Suppose that $\mathcal{L}=\pi^{*} \mathcal{L}^{\prime} \otimes \mathcal{O}(-d)$, where $\mathcal{O}(1)$ is the tautological line bundle of $\pi: P \rightarrow C$ and $\mathcal{L}^{\prime}$ is a line bundle of $C$. We aim to show that $d=0$. 
Since $\varphi$ is a blowup, $\left.\omega_{E} \simeq \varphi^{*} \omega_{X}\right|_{E} \otimes \mathcal{O}_{E}((n+1) E)$ where $n+1=\operatorname{codim}(P, X)$. Let $h=p \circ \pi$, we now calculate $\left.\left.\varphi^{*} \omega_{X}\right|_{E} \simeq p^{*} \omega_{X}\right|_{P}$ as follows:

$$
\left.\varphi^{*} \omega_{X}\right|_{E} \simeq p^{*} \omega_{P} \otimes\left(\operatorname{det} N_{P / X}\right)^{-1} \simeq h^{*} \omega_{C} \otimes p^{*} \mathcal{L}^{\otimes(-n-1)} .
$$

So $\omega_{E} \simeq h^{*} \omega_{C} \otimes p^{*} \mathcal{L}^{\otimes(-n-1)} \otimes \mathcal{O}_{E}((n+1) E)$. For any fiber $F \simeq \mathbb{P}^{n}$ of $p^{+}$, $h(F)=c$ for some point $c \in C$. The morphism $p$ maps $F$ into $\pi^{-1}(c)=\mathbb{P}^{n+1}$ as a hyperplane, which is illustrated as follows:

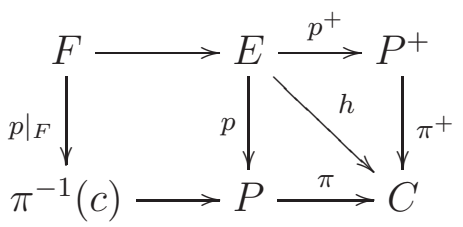

Since $\left.\mathcal{O}_{E}(E)\right|_{F} \simeq \mathcal{O}(-1)$, then we have that

$$
\left.\left\{h^{*} \omega_{C} \otimes p^{*} \mathcal{L}^{\otimes(-n-1)} \otimes \mathcal{O}_{E}((n+1) E)\right\}\right|_{F} \simeq \mathcal{O}((n+1)(d-1)) .
$$

We know that $\left.\omega_{E}\right|_{F} \simeq \mathcal{O}(-n-1)$, so $d=0$ which means that $N_{P / X} \simeq \Omega_{P / C} \otimes$ $\pi^{*} \mathcal{L}^{\prime}$ and $\theta$ is a twisted Mukai flop over $C$.

Note that by Lemma 2.2, we know that there is a lower bound for the dimension of $P$. As an application of Theorem 2.6, we will classify the birational morphism $\theta$ when $\operatorname{dim} P$ reaches the lower bound. Here, what is different from Theorem 2.6, we don't assume that $P$ and $P^{+}$are projective bundles in advance, actually we obtain a result as follows:

Theorem 2.7. (1) If $\operatorname{dim} X=2 \operatorname{dim} P+1$, then $\theta$ is a standard flop.

(2) If $\operatorname{dim} X=2 \operatorname{dim} P$, then $\theta$ is a Mukai flop or a standard flop over a curve.

Proof. (1) If $\operatorname{dim} X=2 \operatorname{dim} P+1$, then $\operatorname{dim} E=2 \operatorname{dim} P$. So the morphism $\left(p, p^{+}\right): E \longrightarrow P \times P^{+}$is surjective. For any fiber $F$ of $p^{+},\left.p\right|_{F}:$ $F \longrightarrow P$ is surjective. By Lazarsfeld's theorem [7], $P$ is a projective space, similarly, $P^{+}$is also a projective space. By Theorem 2.6, $\varphi$ is a standard flop.

(2) If $\operatorname{dim} X=2 \operatorname{dim} P$, then $\operatorname{dim} E=2 \operatorname{dim} P-1$. By Theorem 2 in [13], $E \simeq \mathbb{P}_{P}\left(\Omega_{P}\right)$ where $P$ and $P^{+}$are projective spaces or $E \simeq P \times_{C} P^{+}$ where $C$ is a smooth curve and $P\left(\right.$ resp. $\left.P^{+}\right)$is a projective bundle over $C$. By Theorem 2.6, $\varphi$ is a Mukai flop or a standard flop. 
3. Classifications when $\operatorname{dim} X \leq 5$

Now as an application of all the results we have obtained, we can classify the simple type birational maps when $\operatorname{dim} X \leq 5$. As a first step, we have the following easy corollary from Theorem 2.7.

Corollary 3.1. (1) If $\operatorname{dim} X=3$, then by Lemma 2.2, we have $2 \operatorname{dim} P+$ $1 \geq 3$, so $\operatorname{dim} P=1$. Then $\theta$ is a standard flop by Theorem 2.7.

(2) If $\operatorname{dim} X=4$, then by Lemma 2.2, we have $2 \operatorname{dim} P+1 \geq 4$, so $\operatorname{dim} P=$ 2. Then $\theta$ is a Mukai flop or a standard flop by Theorem 2.7.

(3) If $\operatorname{dim} X=5$, then by Lemma 2.2, we have $2 \operatorname{dim} P+1 \geq 5$, so $\operatorname{dim} P=2$ or 3. If $\operatorname{dim} P=2$, then $\theta$ is a Mukai flop or a standard flop by Theorem 2.7.

In the rest of this section, we keep the assumption that $\operatorname{dim} X=5$. As we see in Corollary 3.1, the remaining unknown case is $\operatorname{dim} P=3$. In this situation, the exceptional divisor $E$ admits two $\mathbb{P}^{1}$ bundle structures as follows:

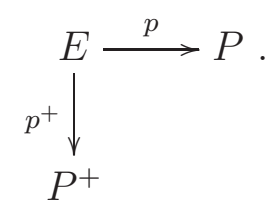

For an arbitrary fibre $F$ of $p^{+}$, it is a natural question to ask whether $p(F)$ is extremal in the cone of curves $\overline{N E(P)}$. Actually, we have the following lemma.

Lemma 3.2. If $E$ admits two $\mathbb{P}^{1}$ bundle structures as above, then $p(F)$ is extremal. Furthermore, the contraction morphism $\pi: P \rightarrow C$ is smooth.

Proof. See Theorem 2.2, [6].

If the picard number $\rho(P) \geq 2$, i.e. $\operatorname{dim} C \geq 1$, we aim to show that $\theta$ is a standard flop or a twisted Mukai flop. First, there exists $\pi^{+}: P^{+} \rightarrow C$ making the following diagram commutative:

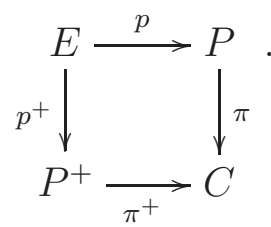

The main obstruction to apply our result Theorem 2.6 is that we don't know, a priori, whether $\pi$ or $\pi^{+}$is a projective bundle. Actually, there is a criterion for projective bundles as follows: 
Lemma 3.3. Suppose that $f: M \rightarrow S$ is a smooth $\mathbb{P}^{k}$ fibration. Let us consider the exact sequence

$$
0 \rightarrow \mathbb{G}_{m} \rightarrow G L_{k+1} \rightarrow P G L_{k} \rightarrow 0,
$$

then we have an exact sequence of étale cohomologies:

$$
H_{e ́ t}^{1}\left(S, G L_{k+1}\right) \stackrel{d}{\rightarrow} H_{e ́ t}^{1}\left(S, P G L_{k}\right) \rightarrow H_{e ́ t}^{2}\left(S, \mathbb{G}_{m}\right),
$$

if $d$ is surjective, then $f$ is a projective bundle. In particular, when $\operatorname{dim} S=1$, $H_{e ́ t}^{2}\left(S, \mathbb{G}_{m}\right)$ vanishes and $d$ is surjective.

Proof. See Theorem 0.1 in [9] and Lemma 1.2 in [10].

Remark 3.4. Note that when $\operatorname{dim} Z \geq 2$, the $H_{e ́ t}^{2}\left(S, \mathbb{G}_{m}\right)$ is not necessarily vanished, things become much more complicated. For example, we consider an arbitrary smooth $\mathbb{P}^{1}$ fibration $f: M \rightarrow S$ which is not a projective bundle. Let $R=M \times_{S} M$, the projection $p: R \rightarrow M$ is always a projective bundle, as the diagonal morphism $\delta: M \rightarrow R$ is a section of $p$ (for details, see Chapter 3, Exercise 4.24 and Chapter $4,[11]$ ). This example shows that the projective bundle structure of $p$ can't decent to $f$, it is the main difficulty in the classification of simple type birational maps when $\operatorname{dim} X=5$.

Lemma 3.5. If $\operatorname{dim} C=1$, then $\theta$ is a twisted Mukai flop over $C$. If $\operatorname{dim} C=2$, then $\theta$ is a standard flop over $C$.

Proof. First, we fix some notations. For any $c \in C$, we let $P_{c}$ be the fibre of $\pi$ and $P_{c}^{+}$be the fibre of $\pi^{+}$. Let $h=\pi \circ p$ and $E_{c}=h^{-1}(c)$. Since $\pi$ is an elementary contraction, the picard numbers $\rho\left(P_{c}\right)=\rho\left(P_{c}^{+}\right)=1$.

We observe that the exceptional divisor $E$ admits two $\mathbb{P}^{1}$ bundle structures over $P$ and $P^{+}$, so there are induced $\mathbb{P}^{1}$ bundle structures of $E_{c}$ over $P_{c}$ and $P_{c}^{+}$.

If $\operatorname{dim} C=1$, then $\operatorname{dim} E_{c}=\operatorname{dim} P_{c}+\operatorname{dim} P_{c}^{+}-1$. By Theorem 2 in [13] and $\rho(P)=1, P_{z}$ and $P_{z}^{+}$are projective spaces. Since $C$ is a curve, $H_{e ̂ t}^{2}\left(C, \mathbb{G}_{m}\right)=0$, then $P$ and $P^{+}$are projective bundles over $C$. Then by Theorem 2.6, $\theta$ is a twisted Mukai flop over $C$.

If $\operatorname{dim} C=2$, then for any $c \in C, \operatorname{dim} P_{c}=\operatorname{dim} P_{c}^{+}=1$. Then $\operatorname{dim} E_{c}=$ $\operatorname{dim} P_{c}+\operatorname{dim} P_{c}^{+}$, by the same argument as in Theorem 2.7, we know that $P_{c}$ and $P_{c}^{+}$are projective lines. So $\pi$ and $\pi^{+}$are smooth $\mathbb{P}^{1}$ fibrations. However, as we show in Remark 3.4, the projective bundle structure of $p$ can't decent to $\pi$. Fortunately, there is a criterion of projective bundles for elementary contractions, see Lemma 3.6 below. Let $l=-P_{c} \cdot K_{P}$. Since $K_{P}=\pi^{*} K_{C} \otimes K_{P / C}, l=2$. 
Then $\pi$ is an elementary contraction of maximal length. By Lemma 3.6, $\pi$ is a projective bundle. By Theorem 2.6, we know that $\theta$ is a standard flop over C.

Lemma 3.6. Suppose that $\pi: P \rightarrow C$ is an elementary contraction of an extremal ray $\Gamma$. Let $l(\Gamma)=\max \left\{K_{P} \cdot A \mid A\right.$ is a rational curve whose numerical class $[A]$ is in $\Gamma\}$. If $\pi$ is an equidimensional fiber contraction of relative dimension $d$ and $l(\Gamma)=-d-1$, then $\pi$ is called an elementary contraction of maximal length and $\pi$ is a projective bundle.

Proof. See Theorem 1.3, [4].

Now we assume that $\rho(P)=1$ i.e. $\operatorname{dim} C=0$. We recall the following result about smooth varieties which admits two $\mathbb{P}^{1}$ bundle structures:

Lemma 3.7. Let $X$ be a complex projective manifold with Picard number $\rho(X)=$ 1 and $\mathcal{E}$ rank 2 vector bundle on $X$. Assume that $Z=\mathbb{P}(\mathcal{E}) \rightarrow X$ admits another smooth morphism $Z \rightarrow Y$ of relative dimension 1 and $n=\operatorname{dim} X \geq 2$. Then,

(1) $X$ and $Y$ are Fano manifolds with $\rho=1$ and there exists a rank 2 vector bundle $\mathcal{E}^{\prime}$ on $Y$ such that $Z \rightarrow Y$ is given by $\mathbb{P}\left(\mathcal{E}^{\prime}\right)$.

(2) if $\mathcal{E}$ and $\mathcal{E}^{\prime}$ are normalised by twisting with line bundles (i.e., $c_{1}=0$ or $-1)$, then $\left((X, \mathcal{E}),\left(Y, \mathcal{E}^{\prime}\right)\right)$ is one of the following, up to exchanging the pairs $(X, \mathcal{E})$ and $\left(Y, \mathcal{E}^{\prime}\right)$ :

(a) $\left(\left(\mathbb{P}^{2}, T_{\mathbb{P}^{2}}\right),\left(\mathbb{P}^{2}, T_{\mathbb{P}^{2}}\right)\right)$, where $T_{\mathbb{P}^{2}}$ is the tangent bundle of the projective plane $\mathbb{P}^{2}$

(b) $\left(\left(\mathbb{P}^{3}, \mathscr{N}\right),\left(Q^{3}, \mathscr{S}\right)\right)$, where $\mathscr{N}$ is a null-correlation bundle on $\mathbb{P}^{3}$ and $\mathscr{S}$ is the restriction to the 3 -dimensional quadric $Q^{3}$ of the universal quotient bundle of the Grassmannian $G\left(1, \mathbb{P}^{3}\right)$,

(c) $\left(\left(Q^{5}, \mathscr{C}\right),\left(K\left(G_{2}\right), \mathscr{L}\right)\right)$, where $\mathscr{C}$ is a Cayley bundle on $Q^{5}, K\left(G_{2}\right)$ is the 5-dimensional Fano homogeneous contact manifold of type $G_{2}$ which is a linear section of the Grassmannian $G\left(1, \mathbb{P}^{6}\right)$ and $\mathscr{L}$ the restriction of the universal quotient bundle on $G\left(1, \mathbb{P}^{6}\right)$.

Proof. See Theorem 1.1, [17].

Now apply the above Lemma 3.7 to our problem, note that $E$ admits two $\mathbb{P}^{1}$ bundle structures and $\operatorname{dim} E=4$, we can assume that $P=\mathbb{P}^{3}$ and the normal bundle $N_{P / X}=\mathscr{N} \otimes \mathcal{O}(d)$ for some integer $d \in \mathbb{Z}$. By the exact sequence for the null-correlation bundle

$$
0 \rightarrow \mathscr{N} \rightarrow T_{\mathbb{P}^{3}}(-1) \rightarrow \mathcal{O}(1) \rightarrow 0
$$


$E$ is a closed subvariety of $\mathbb{P}^{3} \times Q^{3}$ and $p$ is compatible with the projection $\mathbb{P}^{3} \times Q^{3} \rightarrow \mathbb{P}^{3}$ (resp. $p^{+}$is compatible with the projection $\mathbb{P}^{3} \times Q^{3} \rightarrow Q^{3}$, for details, see Proposition 2.6, [16] and Chapter 1, Section 4.2, [12] ).

Example 3.8. Keep the notations as above, we can construct a simple type birational map as follows:

Let $P=\mathbb{P}^{3}$ and the normal bundle $N_{P / X}=\mathscr{N} \otimes \mathcal{O}(d)$. We denote by $\varphi: \widetilde{X} \rightarrow X$ the blowup of $X$ along $P$, hence the exceptional divisor $E$ has a $\mathbb{P}^{1}$ bundle structure over $P$. By Lemma 3.7, $E$ has another $\mathbb{P}^{1}$ bundle structure over a quadric $Q^{3}$, we denote this projective bundle morphism by $p^{+}: E \rightarrow Q^{3}$. Then $\left.\omega_{E} \simeq \varphi^{*} \omega_{X}\right|_{E} \otimes \mathcal{O}_{E}(2 E) \simeq p^{*} \omega_{P} \otimes\left(\operatorname{det} N_{P / X}\right)^{-1} \otimes \mathcal{O}_{E}(2 E)$.

Next we calculate the restriction of $\mathcal{O}_{E}(E)$ on an arbitrary fiber $F$ of $p^{+}$. By Proposition 2.6, [16], $F$ is a projective line in $\mathbb{P}^{3}$ and $c_{1}(\mathscr{N})=0$. So $\left.p^{*} \omega_{P} \otimes\left(\operatorname{det} N_{P / X}\right)^{-1} \otimes \mathcal{O}_{E}(2 E)\right|_{F}=\left.\mathcal{O}(-4-2 d) \otimes \mathcal{O}_{E}(2 E)\right|_{F}$. Since $\left.\omega_{E}\right|_{F} \simeq$ $\left.\omega_{E / Q^{3}}\right|_{F}=\mathcal{O}(-2)$. So $\left.\mathcal{O}_{E}(E)\right|_{F} \simeq \mathcal{O}(d+1)$. By Fujiki-Nakano's criterion(see [2] or Remark 11.10, [5]), there is a blow-down $\varphi^{+}: \widetilde{X} \rightarrow X^{+}$compatible with $p^{+}$ if and only if $d=-2$.

Also, $X^{+}$constructed here is not necessarily projective. As in Remark 1.1, suppose that there exists a flopping contraction $\beta: X \rightarrow \bar{X}$ contracting $P$, then $X^{+}$can be proved projective by the same argument as Proposition 1.3, [8]. For the sake of completeness, we here give a detailed proof as follows: First, $-K_{\tilde{X}} \cdot F=\left.\operatorname{deg}\left(\omega_{E} \otimes \mathcal{O}_{E}(-E)\right)\right|_{F}=-1<0$. So $F$ is a $K_{\tilde{X}}$ negative curve. Next, we aim to show that $[F]$ spans an extremal ray in $\overline{N E(\widetilde{X})}$, i.e., it has a supporting divisor(nef and big). Let $H$ be an ample line bundle on $X$ and $\mathcal{L}=\beta^{*} \bar{H}$, where $\bar{H}$ is an ample line bundle on $\bar{X}$. Then we consider the divisor

$$
\mathcal{L}_{k}^{\prime}=k \varphi^{*} \mathcal{L}-\left(\varphi^{*} H+\lambda E\right)
$$

where $k>>0$ and $\lambda=H \cdot \varphi(F)$. We next show that this divisor is nef and big for large $k$ and vanishes precisely on the ray spanned by $[F]$. For an irreducible curve $A$ in $\widetilde{X}$, we assume firstly that $A \subseteq E$. Since $\rho(E)=2, A$ is numerically equivalent to $a F+b G$ where $G$ is a fiber of $p$ and $a, b$ are positive numbers. Then for any positive $k, \mathcal{L}_{k}^{\prime} \cdot A \geq 0$ and $\mathcal{L}_{k}^{\prime} \cdot A=0$ if and only if $b=0$. Now, suppose that $A \nsubseteq E$, then by the projection formula,

$$
\mathcal{L}_{k}^{\prime} \cdot A=k \mathcal{L} \cdot \varphi(A)-H \cdot \varphi(A)-\lambda E \cdot A=k \bar{H} \cdot \beta(\varphi(A))-H \cdot \varphi(A)-\lambda E \cdot A .
$$


The intersection number with $\bar{H}$, we denote it by $\langle\bar{H}, \cdot\rangle$, defines a linear form on $N_{1}(\bar{X})_{\mathbb{R}}$. Since $\bar{H}$ is ample, there is a positive lower bound of $\langle\bar{H}, \cdot\rangle$ for any compact subset of $\overline{N E(\bar{X})} \backslash\{0\}$. So for large enough $k, \mathcal{L}_{k}^{\prime} \cdot A>0$. In conclusion, for $k>>0, \mathcal{L}_{k}^{\prime}$ is nef and big and vanishes precisely on the ray spanned by $[F]$. Then $F$ is a $K_{\tilde{X}}$ negative extremal curve, so $X^{+}$is projective.

We'd like to thank Will Donovan for pointing out that our Example 3.8 is already known by Roland Aburf, see [15].

In conclusion, we have proved the following theorem.

Theorem 3.9. A birational map of simple type, if $\operatorname{dim} X \leq 5$, is either a standard flop, a twisted Mukai flop or a flop as in Example 3.8.

\section{References}

[1] O.Debarre: Higher-Dimensional Algebraic Geometry. Universitext. Springer-Verlag, New York, 2001

[2] A.Fujiki, S.Nakano: Supplement to "On the inverse of monoidal transformation". Publ.RIMS Kyoto Univ.7(1071), 637-644.

[3] B.Fu, C.-L. Wang: Motivic and quantum invariance under stratified Mukai flops. (English summary) J. Differential Geom. 80 (2008), no. 2, 261-280.

[4] A.Höring, C.Novelli: Mori contraction of maximal length. Publ. Res. Inst. Math. Sci. 49 (2013), no. 1, 215-228.

[5] D.Huybrechts: Fourier-Mukai transforms in algebraic geometry. Oxford Mathematical Monographs. The Clarendon Press, Oxford University Press, Oxford, 2006. viii+307 pp.

[6] A.Kanemitsu: Fano 5-folds with nef tangent bundles.arxiv.org/abs/1503.04579.

[7] R.Lazarsfeld: Some applications of the theory of positive vector bundles. Ann. of Math. 110 (1979) 593-606.

[8] Y.P. Lee, H.-W. Lin, C.-L. Wang: Flops, motives, and invariance of quantum rings. (English summary) Ann. of Math. (2) 172 (2010), no. 1, 243-290.

[9] M.Maruyama: On classification of ruled surfaces. Lectures in Mathematics, Department of Mathematics, Kyoto University, 3 Kinokuniya Book-Store Co., Ltd., Tokyo 1970 iv+75 pp.

[10] M.Maruyama: On a family of algebraic vector bundles. Number theory, algebraic geometry and commutative algebra, in honor of Yasuo Akizuki, pp. 95-146. Kinokuniya, Tokyo, 1973.

[11] James S. Milne: Étale cohomology. Princeton Mathematical Series, 33. Princeton University Press, Princeton, N.J., 1980. xiii+323 pp.

[12] C. Okonek, M. H. Schneider and H. Spindler: Vector bundles on complex projective spaces. Corrected reprint of the 1988 edition. With an appendix by S. I. Gelfand. Modern Birkhäuser Classics. Birkhäuser/Springer Basel AG, Basel, 2011. viii+239 pp.

[13] G.Occhetta, Jarosław A. Wiśniewski: On Euler- Jaczewski sequence and Remmert- Van de Ven problem for toric varieties. Mathematische Zeitschrift, 2001, 241(1):35-44.

[14] E.SATO: Varieties which have two projective space bundle structures. J.Math.Kyoto Univ. 25-3 (1985)445-457. 
[15] Ed Segal: A new 5-fold flop and derived equivalence. Bull. Lond. Math. Soc. 48 (2016), no. 3, 533-538.

[16] M.Szurek; Jarosław A. Wiśniewski: Fano bundles over $P^{3}$ and $Q_{3}$. Pacific J. Math. 141 (1990), no. 1, 197-208.

[17] K.Watanabe: $\mathbb{P}^{1}$-bundles admitting another smooth morphism of relative dimension one. J. Algebra 414 (2014), 105-119.

Address of Duo Li: Yau Mathematical Sciences Center, Tsinghua University, Beijing, 100084, P. R. China.

E-mail address: liduo211@mails.ucas.ac.cn 